\title{
Correlation between Coronary High-Intensity Plaque on T1-Weighted Magnetic Resonance Imaging and Myocardial Injury after Percutaneous Coronary Intervention H.H.Ebaid ${ }^{1}$, A.A.Elgaha ${ }^{2}$, H.S.Abdelrahman ${ }^{3}$, K.E.El Rabbat ${ }^{1}$, M.A.Tabl ${ }^{1}$ and W.S.Abd El Kader ${ }^{1}$ \\ ${ }^{1}$ Cardiovascular, Dept., Faculty of Medicine, Benha Univ., Benha, Egypt \\ ${ }^{2}$ Cardiovascular,Dept.,Faculty of Medicine, Cairo Univ., Cairo, Egypt \\ ${ }^{3}$ Radiology,Dept.,Faculty of Medicine, Ain shams.,Cairo, Egypt \\ Email:
}

\begin{abstract}
Post-procedure myocardial injury/infarction following percutaneous coronary mediation (PCI) is regular in clinical practice and is showed by the rise of heart biomarkers, for example, creatinine kinase or cardiovascular troponin. to show that focused energy plaques on non-contrast T1-weighted MRI imaging may mirror the potential for plaque weakness on IVUS evaluation, and that focused energy plaques may assume a critical part in foreseeing the frequency of postprocedural myocardial injury. This is a forthcoming non-randomized relative investigation was led on 55 patients with ongoing stable angina. Patients were exposed to coronary MRI with non-contrast T1-weighted imaging. They were chosen from cardiovascular medication office, Faculty of Medicine, Benha University, Egypt, Saudi German Hospital, Jeddah, and Saudia Arabia during the time frame from September 2018 till March 2020. There was no huge contrast among IVUS and MRI discoveries with respect to IVUS brings about negative and positive MRI patients $(\mathrm{P}>0.05)$. The affectability and particularity of MRI-HIP in foreseeing moderate were $75 \%$ and $51.16 \%$, separately. Additionally, the affectability and particularity of IVUS low weakening in foreseeing moderate stream were $75 \%$ and $59.53 \%$, separately. Our examination reasoned that, underlying coronary patency upon demonstrative angiography is altogether connected with quick post PCI TIMI stream. Additionally, MRI appraisal of coronary plaques reflecting structure encourages more exact danger delineation and forecast of PMI after elective PCI in stable CAD patients. Further investigations are expected to explain the coronary plaque qualities addressing HIP on non-contrast T1WI.
\end{abstract}

Key words: myocardial injury, PCI, IVUS, MRI.

\section{Introduction}

Post-methodology myocardial injury/ localized necrosis following percutaneous coronary mediation (PCI) is normal in clinical practice and is showed by the height of heart biomarkers, for example, creatinine kinase or cardiovascular troponin. [1] Troponin I height after elective PCI is successive and related with dynamically higher death rates at 1 year. A cutoff estimation of more than or equivalent to multiple times the 99th percentile, presently characterized as myocardial injury, give off an impression of being a considerably more critical indicator of this result, even in lower hazard subgroups. [2]

Atherosclerosis imaging incorporates an armamentarium of set up and trial radiological techniques and modalities. Extensively, these procedures can be utilized to distinguish anatomic and physiological outcomes of long-standing atherosclerosis, to give detail on plaque arrangement and sub-atomic movement, and to appraise biomechanical stresses acting inside the blood vessel framework. Together these strategies give proportions of infection seriousness, which are imperative to regular clinical practice and cardiovascular exploration. [3]

Throughout the decade, intravascular ultrasound (IVUS) has been utilized as an essential obtrusive instrument for coronary plaque appraisal, using its prevalence over coronary aluminography (i.e., coronary angiography). With a pivotal goal of 150 to $250 \mathrm{um}$ and a horizontal goal of $250 \mathrm{um}$, a few discoveries of weakness have been recognized. Notwithstanding traditional dark scale IVUS, new age of IVUS are accessible. [4] Intravascular ultrasound elastography and paleography have additionally been utilized to describe the pressure strain relationship on coronary veins and plaques. Like ultrasound optical portrayal of atherosclerotic plaque may give data about lipid creation of high-hazard plaques. Angioscopy has reliably exhibited a relationship between yellow plaque tone and danger plaques including those causing MI. [5]

Attractive Resonance Imaging (MRI), gives high spatial and transient goal and has the benefit of consolidating anatomic, utilitarian and sub-atomic imaging in an across the board approach. Its delicate tissue contrast empowers to recognize the significant segments of atherosclerotic plaques and permits hazard definitions dependent on plaque creation regardless of the level of stenosis. [6] This planned non-randomized similar preliminary investigation was done to show that extreme focus plaques on non-contrast T1-weighted MRI imaging may mirror the potential for plaque weakness on IVUS appraisal, and that focused energy plaques may assume a critical part in foreseeing the rate of postprocedural myocardial injury.

\section{Patients and Methods}

This is a planned non-randomized similar investigation was led on 55 patients with ongoing stable angina. Patients were exposed to coronary MRI with non-contrast T1-weighted imaging. They were chosen from cardiovascular medication division, Faculty of Medicine, Benha University, Egypt, Saudi German Hospital, Jeddah, and Saudia Arabia during the time frame from September 2018 till March 2020. Among them, three patients who didn't go through PCI and two patients whose picture nature of T1WI was poor (each 
with one coronary vein) were prohibited. Consequently, 76 coronary plaques from 50 patients (76 injuries) were broke down in this examination.

Moral thought: All members were marked a composed educated assent that clarified the point of the investigation before the examination inception, after endorsement of moral board of trustees in Faculty of Medicine, Benha University, Egypt.

All patients remembered for the investigation were chosen by the consideration and prohibition standards:

Incorporation measures: Patients with ongoing coronary syderomes in whom huge coronary supply route stenosis (> 70\%) was analyzed on obtrusive coronary angiography, patients were exposed to coronary MRI with non-contrast T1-weighted imaging, additionally, when patient had in any event one focused energy plaque, he/she was ordered into the focused energy gathering.

\subsection{Exclusion models}

Patients with injury with extreme calcification by a visual gauge of coronary angiogram, Patients with renal debilitation characterized as creatinine of $1.3 \mathrm{mg} / \mathrm{dl}$ or more or glomerular filtration pace of $60 \mathrm{ml} / \mathrm{min}$ or less, Pre-procedural white platelet tallies $9.5 \times 106$ for each L, patients with persistent all out coronary course impediment, patients with past coronary stents or sidestep unites, patients with left fundamental infection and sentence structure score of at least 32, patients with multivessel illness with diabetes, left ventricular brokenness or punctuation score of at least 22, patients with MRI non-viable pacemakers or implantable cardioverter defibrillator and patients with claustrophia.

\subsection{All patients remembered for this examination were exposed to the accompanying}

Full history taking included: individual history, previous history, family ancestry and clinical history taking, Clinical assessment included: Blood pressure, heartbeat, temperature and respiratory rate, research center information Including: Lipid profile, Glycosylated HGB, Renal capacity and complete blood tally. Likewise, Cardiac imaging with echocardiography, CT coronary angiography, single photon outflow tomography or stress echocardiography. Also, coronary attractive reverberation imaging and plaque investigation, As well, Intravascular ultrasound picture securing and examination, Percutaneous coronary mediation methods and estimation of high-touchy cardiovascular troponin-T.

\subsection{Methods}

2.4. Patients were imaged with non-contrast T1weighted imaging inside $48 \mathrm{hrs}$.

Prior to PCI. After procurement of IVUS pictures, the PCI system was performed with the standard procedures. Sequential estimation of exceptionally delicate cardiovascular troponine- $\mathrm{T}$ was performed at both benchmark and $24 \mathrm{hrs}$ after PCI with the point of surveying post procedural myocardial injury, at that point, coronary angiography: When the patient was chosen for percutaneous coronary intercession double antiplatelet consumption with asprin $81 \mathrm{mg} /$ every day and either clopidogrel $75 \mathrm{mg}$ /day by day was guaranteed. Intravascular ultrasound: Equipment: staged cluster test with various fixed transducers with a common sound recurrence of $20 \mathrm{MHz}$ (like Eagle Eye ${ }^{\mathrm{TM}}$, Philips Volcano, USA), Analysis of pictures: intravascular ultrasound quantitative and subjective examinations were acted in blinded way as per the American College of Cardiology Clinical Expert Consensus Document.

\subsection{For bifurcational sores the one of coming up next was finished}

Ordinary stenting of the significant vessel, Classical squash procedure comprises in the sending of the side brach stent. Twofold kissing pound with expansion of a high-pressure expand toward the SB prior to performing kissing inflatable swelling.

\subsection{Statistical Methodology}

Information were gathered, organized, measurably dissected utilizing an IBM PC with Statistical Package of Social Science (SPSS) variant 22 (SPSS, Inc, Chicago, Illinois, USA). Spellbinding information were introduced as mean (X-), standard deviation (SD), range, and subjective information were introduced in the structure numbers and rates. Scientific insights: $\mathrm{t}$ test, Chi-Squared $(\chi 2)$, Pearson connection and ROC (recipient working trademark) bends. Results were viewed as huge if $\mathrm{P} \leq 0.05$ and exceptionally critical if $\mathrm{P} \leq 0.01$.

\section{Results}

The mean age of the included patients was $59.58 \pm 5.97$ years. Majority of the patients $(80 \%)$ were males. The mean BMI was $33.94 \pm 4.66 \mathrm{~kg} / \mathrm{m}^{2}$ and $(38 \%)$ of patients were smokers. Also, $(92 \%)$ of the included patients had hypertension, and (34\%) had DM, while, (40\%) had dyslipidemia. Also, (34\%) of patients had metabolic syndrome, as shown in table 1 .

Table (1) Distribution of the studied patients regarding demographic data, comorbidities.

\begin{tabular}{llcc}
\hline & \multicolumn{2}{c}{ Studied patients } & $\mathbf{N = 5 0}$ \\
\cline { 3 - 4 } & & Mean \pm SD & range \\
\hline Age/ year & $59.58 \pm 5.97$ & $47-72$ \\
BMI $\left(\mathbf{k g} / \mathbf{m}^{\mathbf{2}}\right)$ & $33.94 \pm 4.66$ & $25-46$ \\
\multicolumn{1}{c}{ Sex } & & $\mathrm{N}$ & $\%$ \\
& - Male & $1.2 \pm 0.40$ & $1-2$ \\
- & Female & 40 & $80.0 \%$ \\
\end{tabular}




\begin{tabular}{ccc}
\hline Current Smoker & $0.38 \pm 0.49$ & \\
- Negative & $31(62.0 \%)$ & \\
- Positive & $19(38.0 \%)$ & \\
Multi vessel disease & $0.62 \pm 0.49$ & $1-3$ \\
- Negative & $19(38.0 \%)$ & \\
- Positive & $31(62.0 \%)$ & $1-3$ \\
Co morbidities. & & $8.0 \%$ \\
Hypertension & $0.92 \pm 0.27$ & $92.0 \%$ \\
- Negative & 4 & $1-3$ \\
- Positive & 46 & $66.0 \%$ \\
Diabetes & $0.34 \pm 0.48$ & $34.0 \%$ \\
- Negative & 33 & $1-3$ \\
- Positive & 17 & 60.0 \\
Dyslipidemia & $0.4 \pm 0.49$ & 40.0 \\
- Negative & 30 & $66 \%$ \\
- Positive & 20 & $34 \%$ \\
Metabolic syndrome & $0.34 \pm 0.48$ & \\
- Negative & 33 & \\
\hline
\end{tabular}

Values are mean $+\mathrm{SD}$, or $\mathrm{N}(\%)$.

HTN: Hypertension

While, there was statistically high significant difference between MRI groups regarding IVUS low attenuation plaque. Also, there was statistically significant increase among MRI positive group than MRI negative group regarding PMR and increase of Troponine $(\mathrm{P}<0.05)$. While, there was statistically
HIP: high-intensity plaque, $\quad$ BMI: BMI Index

DM: Diabetes Mellitus

significant increase among MRI negative group than the MRI positive group regarding troponine after PCI. There was high significant correlation between MRI finding and PMR. While, IVUS and measurement of troponine at baseline and after PCI didn't show any significant correlations, as shown in table (2).

Table (2) Correlation between presence of MRI high intensity plaque and HGBA1C, lipid profile, IVUS low attenuation plaque, measurement of troponine and patient's medication.

\begin{tabular}{|c|c|c|c|c|c|}
\hline & \multicolumn{2}{|c|}{ MRI finding } & \multirow[b]{2}{*}{$P$-value } & \multirow[b]{2}{*}{$\mathbf{X}^{2}$} & \multirow[b]{2}{*}{ sig } \\
\hline & $\begin{array}{c}\text { Negative } \\
n=23 \\
\text { Mean } \pm \text { SD }\end{array}$ & $\begin{array}{c}\text { Positive } \\
\mathbf{n}=27 \\
\text { Mean } \pm \text { SD }\end{array}$ & & & \\
\hline HGBA1C & $5.78 \pm 0.69$ & $5.904 \pm 0.89$ & $0.094^{\mathrm{NS}}$ & 0.585 & 0.561 \\
\hline Total cholesterol mg/dl & $159.83 \pm 14.38$ & $166.07 \pm 24.48$ & $0.157^{\mathrm{NS}}$ & 1.075 & 0.288 \\
\hline Triglycerides mg/dl & $124.13 \pm 42.99$ & $123.41 \pm 45.2$ & $0.876^{\mathrm{NS}}$ & 0.058 & 0.954 \\
\hline HDL cholesterol mg/dl & $39.39 \pm 7.73$ & $40.85 \pm 8.17$ & $0.828^{\mathrm{NS}}$ & 0.646 & 0.521 \\
\hline LDL cholesterol mg/dl & $90.74 \pm 22.82$ & $100.06 \pm 21.09$ & $0.960^{\mathrm{NS}}$ & 1.500 & 0.140 \\
\hline IVUS & $0.49 \pm 0.50$ & $76(100.0 \%)$ & $<0.001 *$ & 0.575 & \\
\hline - Negative & $0.05 \pm 0.22$ & $37(94.8 \%)$ & $39(51.3 \%)$ & $<0.000$ & 0.567 \\
\hline - Positive & $0.97 \pm 0.16$ & $2(5.4 \%)$ & $37(48.7 \%)$ & $<0.000$ & \\
\hline PMR & $1.17 \pm 0.24$ & $1.92 \pm 0.37$ & $<0.000^{*}$ & 27.719 & $<0.0001$ \\
\hline \multicolumn{6}{|c|}{ Measurement of troponine } \\
\hline baseline/ ng/mL & $0.03 \pm .097$ & $0.03 \pm 0.09$ & $0.845^{\mathrm{NS}}$ & 0.197 & 0.845 \\
\hline after PCI/ ng/mL & $0.104 \pm .10$ & $0.09 \pm 0.16$ & $<0.031^{*}$ & 0.851 & 0.399 \\
\hline Increase of Troponine & $0.03 \pm 0.05$ & $0.14 \pm 0.12$ & $<0.0001^{*}$ & 4.662 & 0.153 \\
\hline \multicolumn{6}{|c|}{ Patient's medication } \\
\hline Statins & $0.88 \pm 0.326$ & $0.96 \pm 0.204$ & & & \\
\hline - Negative & 3 & 1 & $0.054^{\mathrm{NS}}$ & 0.949 & $0.347^{\mathrm{NS}}$ \\
\hline - Positive & 23 & 23 & & & \\
\hline Beta blocker & $0.65 \pm 0.485$ & $0.71 \pm 0.464$ & & & \\
\hline - Negative & 9 & 7 & $0.421^{\mathrm{NS}}$ & 0.405 & $0.687^{\mathrm{NS}}$ \\
\hline - Positive & 17 & 17 & & & \\
\hline Oral hypoglycemic drugs & $0.23 \pm 0.430$ & $0.42 \pm 0.504$ & & & \\
\hline - Negative & 20 & 14 & $0.166^{\mathrm{NS}}$ & 1.408 & $0.166^{\mathrm{NS}}$ \\
\hline - $\quad$ Positive & 6 & 10 & & & \\
\hline
\end{tabular}


MRI: Magnetic resonance imaging IVUS: Intravascular ultrasound PMR: Plaque to myocardial intensity ratio.

There was statistically significant increase among MRI negative group regarding all parameters except for EEM, P+M, plaque burden, remodeling index, and lipid area as they showed significant increase among MRI positive group $(\mathrm{P}<0.05)$. There were significant correlations between MRI finding and all gray scale IVUS and VH- IVUS parameters except for Positive remodeling ( $\mathrm{p}>0.05)$, as shown in table 3 .

Table (3) Correlation of the presence of MRI high intensity plaque to gray scale IVUS and VH- IVUS parameters.

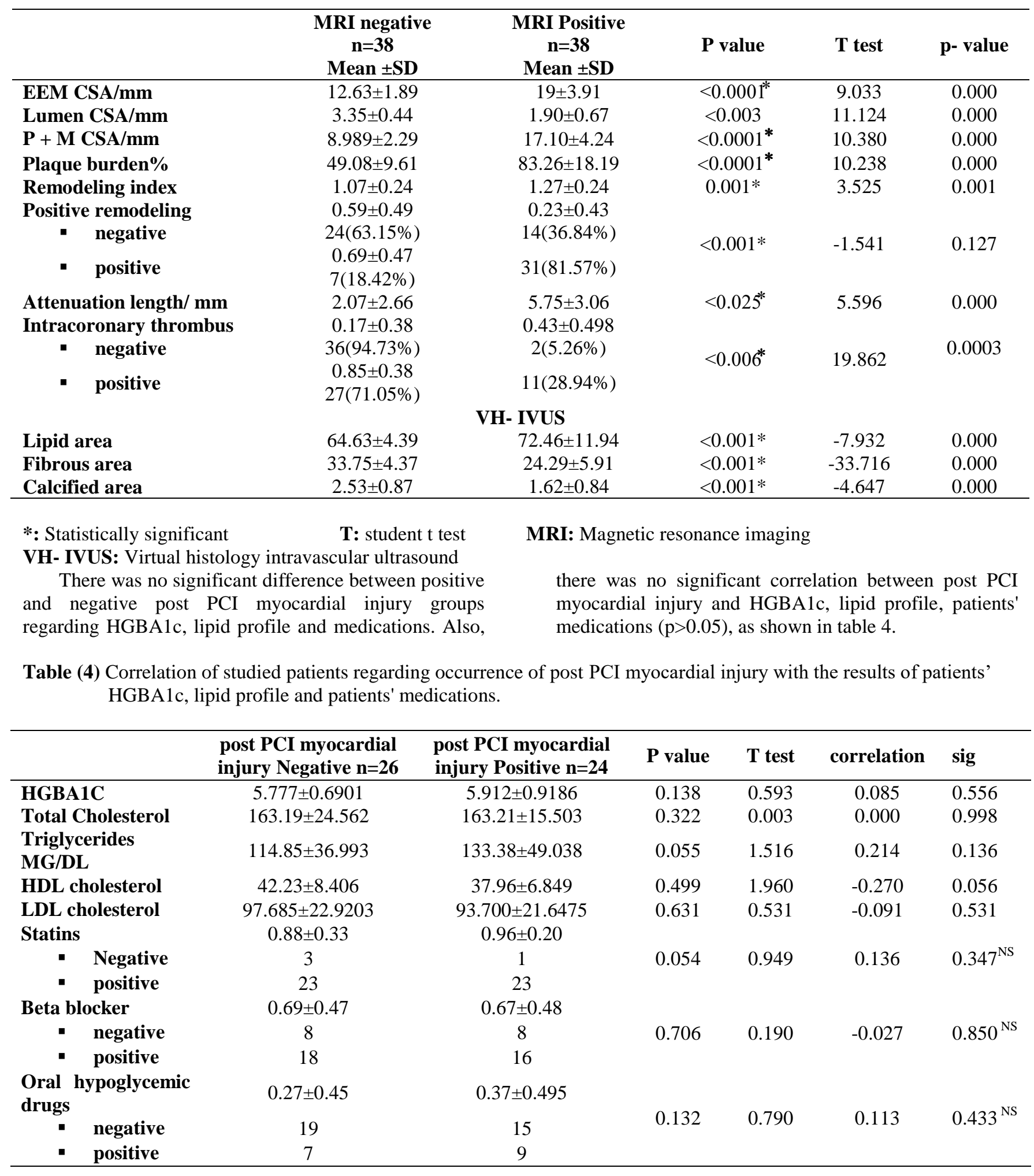


T: student t test Ns: non-significant PCI: Percutaneous coronary intervention HGBA1C: Hemoglobin A1C HDL cholesterol: High density lipoprotein cholesterol LDL cholesterol: Low density lipoprotein cholesterol.

There was significant difference between positive and negative MRI high intensity plaque groups regarding occurrence of PCI related myocardial injury $\mathrm{p}<0.05$. while, there was no significant correlation between them, as shown in table 5 .

Table (5) Correlation of the presence of coronary MRI high intensity plaque and occurrence of PCI related myocardial injury.

\begin{tabular}{|c|c|c|c|c|c|}
\hline & MRI negative Mean \pm SD & MRI Positive mean \pm SD & P value & T test & sig \\
\hline PCI related Myocardial Injury & $0.7 \pm 0.46$ & & & & \\
\hline - Negative & $\begin{array}{l}0.84 \pm 0.37 \\
11(44 \%)\end{array}$ & $5(20 \%)$ & $<0.031^{*}$ & 1.807 & 0.077 \\
\hline - Positive & $\begin{array}{l}0.56 \pm .51 \\
15(60 \%)\end{array}$ & $19(76 \%)$ & & & \\
\hline
\end{tabular}

*: Statistically significant $\quad$ T: student t test $\quad$ PCI: Percutaneous coronary intervention

There was no critical distinction among IVUS and MRI discoveries with respect to IVUS brings about negative and positive MRI patients $(\mathrm{P}>0.05)$. The affectability and particularity of MRI-HIP in anticipating moderate were $75 \%$ and $51.16 \%$, individually with positive and negative prescient estimations of $71.1 \%$, and $91.7 \%$, separately. Likewise, the affectability and particularity of IVUS low lessening in foreseeing moderate stream were $75 \%$ and $59.53 \%$, individually with positive and negative prescient estimations of $48.8 \%$, and $89.5 \%$, separately, as demonstrated in table 6 .

Table (6) Correlation of the detection of coronary high intensity plaques as detected by MRI T1 weighted imaging and the presence of low attenuation plaques in IVUS imaging.

\begin{tabular}{llcccc}
\hline MRI & \multicolumn{2}{c}{ IVUS } & Positive \\
\cline { 2 - 5 } & Negative & N=32 & $\mathbf{X}^{\mathbf{2}}$ & sig \\
\hline - & Negative & 6 & 18 & 2.424 & $0.119^{\text {NS }}$ \\
- & Nositive & 12 & 14 & 0.500 & $0.479^{\text {NS }}$ \\
- & Positive & 16 & 26 & 6 & \\
\hline
\end{tabular}

$\mathbf{X}^{2}$ : Chi square Ns: non-significant

Table (7) Diagnostic accuracy of MRI-HIP in detecting vulnerable coronary plaque and predicting post PCI myocardial injury and slow flow in comparison with IVUS.

\begin{tabular}{|c|c|c|c|c|c|c|c|}
\hline & $\mathbf{A U C}$ & P-value & sensitivity & specificity & PPV & NPV & $95 \% \mathrm{CI}$ \\
\hline $\begin{array}{l}\text { MRI-HIP in detecting } \\
\text { vulnerable coronary plaque } \\
\text { in comparison with IVUS }\end{array}$ & 0.810 & 0.045 & $74.29 \%$ & $87.8 \%$ & $80 \%$ & $83.9 \%$ & $(70.4-0.89 .1)$ \\
\hline $\begin{array}{l}\text { MRI high intensty plaque in } \\
\text { predicting slow flow }\end{array}$ & & & $75 \%$ & $51.16 \%$ & 71.1 & 91.7 & $\begin{array}{c}26.16 \\
(1.16-55.57)\end{array}$ \\
\hline $\begin{array}{l}\text { IVUS low attenuation in } \\
\text { predicting } \\
\text { Slow flow }\end{array}$ & & & $75 \%$ & $59.53 \%$ & 48.8 & 89.5 & $\begin{array}{c}14.53 \\
(0.29-40.99)\end{array}$ \\
\hline
\end{tabular}

MRI-HIP: Magnetic resonance imaging high intensity plaque

IVUS: Intravascular ultrasound PCI: Percutaneous coronary intervention.

\section{Discussion \\ This forthcoming non-randomized relative} preliminary investigation was done to exhibit that focused energy plaques on non-contrast T1-weighted MRI imaging may mirror the potential for plaque weakness on IVUS appraisal, and that focused energy plaques may assume a crucial part in foreseeing the occurrence of postprocedural myocardial injury. The investigation was led on 55 patients with persistent coronary conditions. Patients were exposed to coronary MRI with non-contrast T1-weighted imaging. They were chosen from cardiovascular medication division, Faculty 
of Medicine, Benha University, Egypt, Saudi German Hospital, Jeddah, and Saudia Arabia during the time frame from September 2018 till March 2020.

The current examination showed that the mean age of the included patients was 59.58 \pm 5.97 years. Dominant part of the patients $(80 \%)$ were guys. The mean BMI was $33.94 \pm 4.66 \mathrm{~kg} / \mathrm{m} 2$ and $(38 \%)$ of patients were smokers. Likewise, (92\%) of the included patients had hypertension, and (34\%) had DM, while, (40\%) had dyslipidemia. Likewise, (34\%) of patients had metabolic disorder. There was no huge connection between's MRI finding and all segment information and comorbidities. This in line with [7] who found no huge contrasts among HIP and no HIP gatherings in the attributes (age, sex, BMI, Hypertension, Diabetes mellitus, Dyslipidemia and Current smoker).

The current investigation likewise showed that, there was no measurably critical contrast among negative and positive MRI finding with respect to HGBA1C, all out cholesterol, fatty oils, HDL and LDL cholesterol. Our outcomes showed no huge contrast among positive and negative MRI discoveries in regards to patient's drugs, aside from metformin, GLRA, DPP4I and thiazolidinedione's which showed measurably critical increment among positive MRI finding than negative MRI finding. In the equivalent line [7] found no genuinely critical contrast among HIP and HIP gatherings with respect to Medications (Aspirin, Clopidogrel, Statin, Beta-blocker, ACEI and additionally $\mathrm{ARB}$ and Calcium channel blocker). The relationship with the already mensioned medications can clarified by the presence diabetes as opposed to the medications which likewise coordinates with a deliberate investigation of 2,237 subjects from randomized controlled examinations assessing plaque movement because of different pharmacological treatments utilizing sequential IVUS, the degree of coronary atherosclerosis, example of blood vessel renovating and infection movement was thought about among DM and non-DM patients. It was seen that DM subjects had more broad atherosclerosis and complete atheroma volume (TAV) when contrasted with non-DM subjects (199.4 \pm 7.9 versus $189.4 \pm 7.1 \mathrm{~mm} 3, \mathrm{P}=0.03)$. In spite of the presence of more broad infection, DM subjects had more modest lumen and comparable outer flexible film (EEM) representing more prominent percent atheroma volume (PAV) [(40.2 \pm 0.9$) \%$ versus $(37.5 \pm 0.8) \%, \mathrm{P}<0.0001]$.

Bifurcation sores are especially hard to evaluate by angiography alone on the grounds that covering side branches frequently dark the injury [8] In our examination, there was genuinely critical relationship between's quality of focused energy plaque in MRI and bifurcation injuries, number of influenced vessels. This concurred with [7] found no huge contrasts in regards to add up to cholesterol, fatty oil, HDL cholesterol, LDL cholesterol and Hemoglobin A1c. They showed that the lipid pool was fundamentally more prominent in the patients with HIP dependent on IB-IVUS appraisal. These IVUS discoveries would produce that HIP is relating with the qualities of weak plaque. Likewise, they theorize that coronary plaque with HIP on non-contrast T1WI may address weakness, including parts of an intraplaque discharge, a lipid-rich necrotic center, a clots arrangement, or a combination of these. Notwithstanding, the genuine reason for high-signal force in atherosclerotic coronary plaque on T1WI stays being talked about. Another examination by [9] uncovered that, lipid-rich necrotic carotid plaque shows a focused energy signal on T1WI successions, since intraplaque discharge regularly happens inside lipid-rich necrotic centers. Ongoing case report portrayed that coronary suctioned example with HIP on T1WI shows a lot of the necrotic center with overlying platelet and fibrin-rich clots [10]. Extra discoveries by [11] found that in interwall HIS sores the presence of lipid-rich plaques was more continuous than in non-HIS injuries, albeit these distinctions were not genuinely huge when broke down by multivariate examination [12] Therefore, it isn't known whether the lipids inside the atherosclerotic plaques were effectively smothered. There is expanding proof that various weak plaques with lipid are available inside the entire coronary tree in patients who experience an ACS, despite the fact that it very well might be a solitary restricted offender injury that caused the intense cardiovascular occasion. Be that as it may, most lipid pools don't produce HISs besides at the offender injury. The extent, age, and volume of methemoglobin dependent on the presence of weak complex plaques may decide the PMR esteems [13]

A couple of clinical examinations have analyzed coronary plaque MRI on non-balance T1WI with MDCT, IVUS, and OCT. In our investigation, there was measurably critical relationship between's MRI finding with dark scale IVUS and VH-IVUS boundaries.

The current discoveries uncovered that, affectability and explicitness of MRI-HIP for identifying weak coronary plaques characterized as low weakening plaque with IVUS were as $74.29 \%$ and $87.8 \%$, separately. This matches with what had been distributed by [7] discovered high genuinely huge distinction among HIP and NO HIP gatherings in regards to Gray-scale IVUS boundary (EEM CSA, P + M CSA, Plaque trouble, \%, Remodeling record, Positive rebuilding, Ultrasound weakening, Attenuation length, Intracoronary clots, Lipid zone and, Fibrous region, EEM volume, $\mathrm{P}+\mathrm{M}$ volume, Plaque trouble, \%, Lipid volume). while, no measurably huge contrast was found with respect to (Lumen CSA, Calcified territory and Lumen volume, Calcified volume). They picked to utilize the meaning of HIP as coronary plaques with a PMR of $\geq 1.4$ in this examination. On this premise, they identified HIP in $36 \%$ of the coronary plaques in patients with stable angina pectoris. They appeared with IVUS evaluation that HIP was altogether connected with ultrasound constriction and positive renovating.

In examination with MDCT and MR pictures of the coronary veins, HIP on non-balance T1WI was related with a high recurrence of plaque with low CT thickness (13), (14) detailed a normal insignificant CT thickness of 223.2+20.7 Hounsfield units (HU) in HIP injuries, yet 
9.6+20.5 HU in non-HIP sores. They likewise showed that coronary HIP was related with ultrasound constriction and vessel positive redesigning by dark scale IVUS. In accordance with our discoveries, [11] showed that HIP was related with intracoronary clots on OCT. Alluding to these past investigations, [15] and [11] coronary plaque with a PMR of.1.0 was characterized to be positive for HIP. [16] showed that the presence of HIP (plaques with a PMR of $\geq 1.4$ ) on non-contrast T1WI is altogether connected with future coronary occasions. Then again, [17] showed that patients with higher 3DiPMR had positive vessel renovating contrasted and patients with lower 3Di-PMR, and patients with higher 2D-PMR had longer weakening length contrasted and patients with lower 2D-PMR. Since coronary plaque volume and structure assume a significant part in PMI after elective PCI, the current 3D assessment utilizing non-contrast $\mathrm{T} 1 \mathrm{w}$ imaging for coronary atherosclerosis may improve the exactness of anticipating cardiovascular occasions. To diminish the frequency of pMI and improve clinical results, this noninvasive preoperative assessment before planned PCI may encourage hazard separation for subgroups at high danger for pMI, who may then profit by concentrated pharmacological methodologies (e.g., statins and antiplatelet treatment) and utilization of channel gadgets during elective PCI systems. [18, 19, 20]

With any imaging method, its most significant characteristics are the spatial goal needed to picture the sore segments and great differentiation between the different parts of the injuries. A few agents have detailed that coronary corridor HISs on T1WI is related with a weak plaque morphology and an expanded danger of future cardiovascular occasions, [16, 21,22]. The current investigation exhibited a high measurably huge expansion in presence of focused energy plaque identified with MRI. This is steady with [23] and [24] uncovered that, MR imaging innovation has arrived at an adequate degree of spatial goal, which permitted the plaque perception of huge and static corridors, for example, the carotids and aorta. The appearance of carotid plaque portrayal with non-contrast T1-weighted imaging (T1WI) in MR has encouraged plaque imaging dependent on the presence of an extreme focus signal (HIS) inside the blood clot or intraplaque drain brought about by methemoglobin $\mathrm{T} 1$ shortening, $[25,26]$. In a similar setting, [11] study, coronary plaque pictures have been gotten while the patients were breathing uninhibitedly, by utilizing a three-dimensional T1WI, reversal recuperation, inclination reverberation procedure with fat-concealment. In their examination including few patients, they exhibited an immediate relationship between coronary HISs on T1WI and the presence of intracoronary blood clot as distinguished through OCT [15] revealed that the ordinary coronary HIS on T1WI was related with a high recurrence of IVUS-inferred low constriction and positive redesigning, strikingly low CT thickness, and transient lethargic stream marvels during percutaneous coronary intercession (PCI). These highlights appeared to address weak plaques. Also, [22] detailed that the HIS on T1WI accurately compared to the intracoronary blood clot recognized by intrusive coronary angiography in patients with intense myocardial dead tissue inside $72 \mathrm{~h}$ after the underlying o initiation. [30]

\section{Conclusion}

Our study concluded that, initial coronary patency upon diagnostic angiography is significantly associated with immediate post PCI TIMI flow. Also, MRI assessment of coronary plaques reflecting composition facilitates more accurate risk stratification and prediction of PMI after elective PCI in stable CAD patients. Further studies are needed to clarify the coronary plaque characteristics representing HIP on non-contrast T1WI.

\section{References}

[1] BR.Chaitman, KP.Alexander, DD.Cyr . Myocardial Infarction in the ISCHEMIA Trial: Impact of Different Definitions on Incidence, Prognosis, and Treatment Comparisons. Circulation.vol.8,pp.99111,2020 .

[2] JL.Januzzi, SA.Mahler, RH.Christenson. Recommendations for institutions transitioning to high-sensitivity troponin testing: JACC Scientific Expert Panel. Journal of the American College of Cardiology.vol.73(9),pp.1059-77,2019.

[3] AA.Mahabadi, C.Rischpler. Cardiovascular imaging in cardio-oncology. Journal of thoracic disease. Dec.vol.10(Suppl 35),pp.S4351,2018.

[4] L.Li, Y.Jia, J.Fan. Sodium-fluoride PET-CT for the non-invasive evaluation of coronary plaques in symptomatic patients with coronary artery disease: a cross-correlation study with intravascular ultrasound. European journal of nuclear medicine and molecular imaging.vol.45(12),pp.2181-9,2018.

[5] CC.Shih, PY.Chen, T.Ma . Development of an intravascular ultrasound elastography based on a dual-element transducer. Royal Society open science.vol.5(4),pp.180138,2018.

[6] D.Leithner, L.Moy, EA.Morris. Abbreviated MRI of the breast: does it provide value. Journal of Magnetic Resonance Imaging.vol.49(7),pp.e85100,2019 .

[7] T.Hoshi, A.Sato, D.Akiyama, D.Hiraya, S.Sakai, M.Shindo, K.Mori, M.Minami, K. Aonuma. Coronary high-intensity plaque on $\mathrm{T}$ 1-weighted magnetic resonance imaging and its association with myocardial injury after percutaneous coronary intervention. European heart journal. Aug.vol.1;36(29),pp.1913-22,2015.

[8] S.Mehrotra, S.Mishra, G.Paramasivam. Imaging during percutaneous coronary intervention for optimizing outcomes. Indian heart journal. Dec.vol.1;70,pp. S456-65,2018.

[9] Y.Watanabe, M.Nagayama. MR plaque imaging of the carotid artery. Neuroradiology. Apr.vol. 1;52(4),pp.253-74,2010.

[10] Y.Asaumi, T.Noguchi, Y.Morita, TA.Matsuyama, F.Otsuka, R.Fujiwara, T.Kanaya, 
T. Nagai, M.Higashi, K.Kusano, T.Anzai. NonContrast T1-Weighted Magnetic Resonance Imaging at 3.0 Tesla in a Patient Undergoing Elective Percutaneous Coronary InterventionClinical and Pathological Significance of HighIntensity Plaque-. Circulation Journal. Dec .vol.25;79(1),pp.218-20,2014.

[11] S.Ehara, K.Matsumoto, K.Shimada. The clinical value of high-intensity signals on the coronary atherosclerotic plaques: noncontrast $\mathrm{T} 1$-weighted magnetic resonance imaging. International journal of molecular sciences.Jul.vol.17(7),pp.1187,2016.

[12] K.Matsumoto, S.Ehara, T.Hasegawa, M.Sakaguchi, K.Otsuka, J.Yoshikawa, K. Shimada. Localization of coronary high-intensity signals on T1-weighted MR imaging: relation to plaque morphology and clinical severity of angina pectoris. JACC: Cardiovascular Imaging. Oct.vol.8(10),pp.1143-52,2015.

[13] T.Kubo, T.Imanishi, M.Kashiwagi, H.Ikejima, H.Tsujioka, A.Kuroi, K.Ishibashi, K. Komukai, T.Tanimoto, Y.Ino, H.Kitabata. Multiple coronary lesion instability in patients with acute myocardial infarction as determined by optical coherence tomography. The American journal of cardiology. Feb.vol.1;105(3),pp.318-22,2010.

[14] D.Maintz, M.Ozgun, A.Hoffmeier, R.Fischbach, WY.Kim, M.Stuber, WJ.Manning, W. Heindel, RM. Botnar. Selective coronary artery plaque visualization and differentiation by contrastenhanced inversion prepared MRI. European heart journal. Jul.vol. 1,27(14),pp.1732-6,2006.

[15] T.Kawasaki, S.Koga, N.Koga, T.Noguchi, H.Tanaka, H.Koga, T.Serikawa, Y.Orita. Characterization of hyperintense plaque with noncontrast T1-weighted cardiac magnetic resonance coronary plaque imaging: comparison with multislice computed tomography and intravascular ultrasound. JACC: Cardiovascular Imaging. Jun.vol.2(6),pp.720-8,2009.

[16] T.Noguchi, T.Kawasaki, A.Tanaka, S.Yasuda, Y.Goto, M.Ishihara, K.Nishimura, Y. Miyamoto, K. Node, N.Koga High-intensity signals in coronary plaques on noncontrast T1-weighted magnetic resonance imaging as a novel determinant of coronary events. Journal of the American College of Cardiology. Mar.vol. 18.63(10),pp.989-99,2014.

[17] H.Hosoda, Y.Asaumi, T.Noguchi, Y.Morita, Y. Kataoka. Three-dimensional assessment of coronary high-intensity plaques with T1-weighted cardiovascular magnetic resonance imaging to predict periprocedural myocardial injury after elective percutaneous coronary intervention. Journal of Cardiovascular Magnetic Resonance. Dec.vol. 22(1),pp.1-1,2020.

[18] GW.Stone, A.Maehara, AJ.Lansky, B.De Bruyne, E.Cristea, GS.Mintz, R.Mehran. prospective natural-history study of coronary atherosclerosis. New England journal of medicine. Jan.vol. 20,pp.364(3):226-35,2011.
[19] S.Basili, G.Tanzilli, V.Raparelli, C.Calvieri, P.Pignatelli, R.Carnevale, M.Dominici, A. Placanica, A.Arrivi, A.Farcomeni, F.Barillà. Aspirin reload before elective percutaneous coronary intervention: impact on serum thromboxane b2 and myocardial reperfusion indexes. Circulation: Cardiovascular Interventions. Aug.vol.7(4),pp.577-84,2014.

[20] BE.Claessen, A.Maehara, M.Fahy, K.Xu, GW.Stone, GS.Mintz. Plaque composition by intravascular ultrasound and distal embolization after percutaneous coronary intervention. Cardiovascular Imaging. Mar.vol. 1;5(3 Supplement S),pp.S111-8,2012.

[21] S.Ehara, T.Hasegawa, S.Nakata, K.Matsumoto, S.Nishimura, T.Iguchi, T.Kataoka, J. Yoshikawa, M. Yoshiyama. Hyperintense plaque identified by magnetic resonance imaging relates to intracoronary thrombus as detected by optical coherence tomography in patients with angina pectoris. European Heart Journal-Cardiovascular Imaging. May 1.vol.13(5),pp.394-9,2012.

[22] CH.Jansen, D.Perera, MR.Makowski, AJ.Wiethoff, A.Phinikaridou, RM.Razavi, MS. Marber, GF. Greil, E. Nagel, D.Maintz, S. Redwood. Detection of intracoronary thrombus by magnetic resonance imaging in patients with acute myocardial infarction. Circulation. Jul 26.vol.124(4),pp.416-24,2011.

[23] TS.Hatsukami, R.Ross, NL.Polissar, C.Yuan. Visualization of fibrous cap thickness and rupture in human atherosclerotic carotid plaque in vivo with high-resolution magnetic resonance imaging. Circulation. Aug.vol. 29;102(9),pp.959-64,2000.

[24] AR.Moody, RE.Murphy, PS.Morgan, AL. Martel, GS. Delay, S.Allder, ST.MacSweeney, WG.Tennant, J.Gladman, J.Lowe, BJ.Hunt. Characterization of complicated carotid plaque with magnetic resonance direct thrombus imaging in patients with cerebral ischemia. Circulation. Jun.vol.24;107(24),pp.3047-52,2003.

[25] H.Sinclair, C.Bourantas, A.Bagnall, GS.Mintz, V.Kunadian. OCT for the identification of vulnerable plaque in acute coronary syndrome. JACC: Cardiovascular Imaging. Feb.vol.8(2),pp.198-209,2015.

[26] AV.Finn, M.Nakano, J.Narula, FD.Kolodgie, R.Virmani. Concept of vulnerable/unstable plaque. Arteriosclerosis, thrombosis, and vascular biology. Jul.vol. 1;30(7),pp.1282-92,2010.

[27] Y.Asaumi, T.Noguchi, Y.Morita, R.Fujiwara, T.Kanaya, TA.Matsuyama, T.Kawasaki, M.Fujino, T.Yamane, T.Nagai, T.Miyagi. High-intensity plaques on noncontrast T1-weighted imaging as a predictor of periprocedural myocardial injury. JACC: Cardiovascular Imaging. Jun.vol.8(6),pp.741-3,2015.

[28] S.Kaul. The "no reflow" phenomenon following acute myocardial infarction: mechanisms and 
treatment options. Journal of cardiology. Aug 1.vol.64(2),pp.77-85,2014.

[29] RV.Milani, R.Fitzgerald, JN.Milani, CJ. Lavie. The impact of micro troponin leak on long-term outcomes following elective percutaneous coronary intervention. Catheterization and Cardiovascular Interventions. Nov 15.vol.74(6),pp.819-22,2009.

[30] DN.Feldman, L.Kim, AG.Rene, RM.Minutello, G.Bergman, SC.Wong. Prognostic value of cardiac troponin-I or troponin-T elevation following nonemergent percutaneous coronary intervention: A meta-analysis. Catheterization and Cardiovascular Interventions. Jun .vol.1;77(7),pp.1020-30,2011.

[31] K.Matsumoto, S.Ehara, T.Hasegawa, K.Otsuka, J.Yoshikawa, K.Shimada. Prediction of the filter no-reflow phenomenon in patients with angina pectoris by using multimodality: magnetic resonance imaging, optical coherence tomography, and serum biomarkers. Journal of cardiology. May.vol. 1,pp.67(5):430-6,2016.

[32] SJ.Nicholls, S.Fernando, CA.Bursill . Pathophysiology of Atherosclerosis. InMechanisms of Vascular Disease.vol.8,pp. 19-45,2011.

[33] Y.Kataoka, H.Hosoda, Y.Asaumi, T.Noguchi, Y.Morita, F.Otsuka, K.Nakao, M.Fujino, T.Nagai, M.Nakai, K.Nishimura. Three-dimensional assessment of coronary high-intensity plaques with
T1-weighted cardiovascular magnetic resonance imaging to predict periprocedural myocardial injury after elective percutaneous coronary intervention. Journal of Cardiovascular Magnetic Resonance. Dec.vol.22(1),pp.1-1,2014.

[34] C.Rafanelli, S.Gostoli, S.Buzzichelli. Sequential Combination of Cognitive-Behavioral Treatment and Well-Being Therapy in Depressed Patients with Acute Coronary Syndromes: A Randomized Controlled Trial (TREATED-ACS Study). Psychotherapy and Psychosomatics.vol.89(6),pp.345-56,2020.

[35] A.Arbab-Zadeh, V.Fuster. From detecting the vulnerable plaque to managing the vulnerable patient: JACC state-of-the-art review. Journal of the American College of Cardiology. Sep 24.vol.74(12),pp.1582-93,2019.

[36] AS.Bäck, MS.Hansson. Specialized proresolving Lipoprotein-associated phospholipase A2 in cardiovascular disease, diagnosis, and therapy. Advanced Drug Delivery Reviews.vol.7,pp.44-125, 2015.

[37] JB.Kytö. An overview of near-infrared spectroscopy (NIRS) for the detection of insect pests in stored grains. Journal of Stored Products Research.vol. 86,pp. 101558,2019. 